\title{
AUTOMATIC DEFORMATION INSPECTION METHOD FOR DIGITAL AERIAL IMAGERY BASED ON STATISTICAL CHARACTERISTICS
}

\author{
YI Yaohua ${ }^{1, a}$, Yuan Yuan ${ }^{1, b}$, SU Hai ${ }^{1}$, MIAO Mingjing ${ }^{1}$ \\ ${ }^{1}$ School of Printing and Packaging, Wuhan University \\ 129\# Luoyu Road, Wuhan, China \\ ayyh@whu.edu.cn, byuany@whu.edu.cn
}

KEY WORDS: Digital Aerial Imagery; Imagery Deformation; IMU Data Accuracy; Automatic Deformation Inspection, Statistical Characteristics; Cubic Spline Curve

\begin{abstract}
:
The geometric distortion of the push-broom digital aerial imagery can be rectified according to the data of the inertial measurement unit (IMU). The low precision of IMU data will cause the undulant wavelike twist deformations of the push-broom digital aerial images after geometric rectification, directly influencing the authenticity and liability of images and their practical applications. At present, the image deformation diagnosis mainly depends on the subjective judgment of human being, which costs much time and manpower. In the paper, an automatic deformation inspection method based on statistical characteristics for digital aerial imagery is proposed to inspect the distortion caused by the low IMU data accuracy. For the undulant wavelike deformation image has the characteristic of pixel displacement in the regularly same direction, there will be a lot of wave curves in the same direction appeared in the image after geometric correction. Therefore, in the method, the positions of the wave curves in the image will be located by the extreme points of curvature of the contour lines, and then the wavelike deformations can be judged automatically through the distribution statistics of the open directions of the wave curves. The specific implement method can be described as follows: firstly, the edges of the image are detected with Canny edge detector and the vector contour lines are obtained by tracing the edges to get contour lines and fitting them with the cubic spline curve method. Then, the extreme points of curvature of the contour lines are calculated, and some of these points are determined to be the vertexes of the wave curves by judging the positional relations between each extreme point and the points around it, thus constituting a vertex set. Afterwards, the perpendicular directions of the tangent of the vertexes are used as the directions of the wave curves, and then the direction histograms of all the wave curves in the image are obtained by statistical analysis. Finally, the existence of the deformation phenomenon in the image due to the low precision of IMU data is able to be judged based on whether the directions of the wave curves are centralized in a certain direction or not. Experimental results showed that the automatic deformation inspection method presented in this paper can detect the deformation of the digital aerial images effectively caused by low accuracy of IMU data with $95 \%$ accuracy.
\end{abstract}

\section{GENERAL INSTRUCTIONS}

With the technological progress and widely practical application, the technology of POS(GPS/IMU)-based aerial photogrammetry is becoming more mature and is gradually applied to each field of aerial remote sensing. The geometric distortion of the imagery can be corrected through the use of POS data in this technology. The IMU data of poor accuracy is always processed by linear interpolation calculation to satisfy the requirement of geometric calibration. However, the interpolation error will result in the regional pixel displacement in the same direction of the imagery after geometric rectification. Such distortion phenomenon will severely influence the geometric accuracy of the digital aerial images and their normal use.

In practical applications, it often requires to inspect the deformation of the aerial images without POS data. For the deformations caused by low precision of IMU data, the definition of the aerial imagery is usually computed to detect the existence of image deformation. Yet, the definition won't have a significant reduction only when the deformation phenomenon of the imagery is particularly strong. In other words, the common distortions are not sensitive enough to the definition of the aerial imagery. Simultaneously, the quality of the imagery itself will also affect the definition to some extent. Therefore, as a detection index, the definition is of less use to inspect the deformation because of the limited precision. It still lacks an effective method in the relevant literatures both at home and abroad in terms of the deformation inspection method for digital aerial imagery.
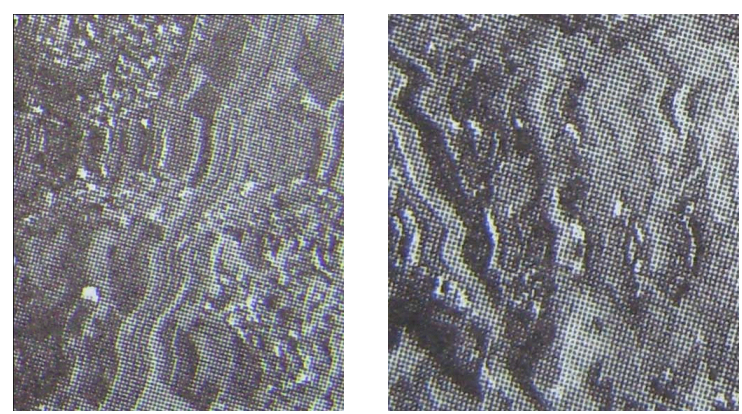

Figure 1. schematic of the image distortion caused by poor accuracy of IMU data

\section{DEFORMATION INSPECTION METHOD FOR DIGITAL AERIAL IMAGERY BASED ON STATISTICAL CHARACTERISTICS}

In the paper, the curve segments of the texture contours approximating a parabolic section in the imagery are defined as squiggles, and the opening directions of the squiggles act as their directions. In addition, the deformation phenomenon of the

\footnotetext{
* YI Yaohua, 1976, professor, Ph.D supervisor, majors in digital color imaging.
} 
imagery described in this paper all refers to that resulting from the poor accuracy of IMU data.

As illustrated above, the insufficient precision of IMU data will give rise to the pixel displacement in the regularly same direction in part of the aerial images, and noticeable wavelike deformations will arise in the edges of the images in the corresponding directions. Although there are also some texture contours approximating squiggles in normal images, the aerophotographs record the ground information and their texture contours are usually without regularity. As a result, the directions of the squiggles are uniformly distributed in all directions instead of gathering in certain direction. Therefore, it is available to inspect the deformation phenomenon through the statistics of directions of the squiggles and constructing a histogram, which is also the main idea of this paper.

The core problem of constructing the histogram of directions is to find out squiggles from the complex digital aerial images. As for searching and matching a specified graphic or template, the common methods include hough transfom, template matching, invariant moments matching, etc. However, since the squiggles of push-broom digital aerial images lacking of sufficient IMU precision have various shapes, it is difficult to describe functions and establish template for them, hence the matching methods are not applicable and suitable.

Through observation, most squiggles are found to be similar to parabola. The approximate vertexes are basically located in the minimum curvature points, and the corresponding directions are consistent with the normal directions of the vertexes. Thus, the positions of the squiggles can be found out by searching the extreme points of curvature of the contour lines in the image and judging whether they are the vertexes of the squiggles. Afterwards, the normal directions of the vertexes of the squiggles are calculated and the distributions of directions are obtained through statistical analysis. Finally, the deformation of the aerial imagery can be determined. The basic workflow is shown in figure 2 .

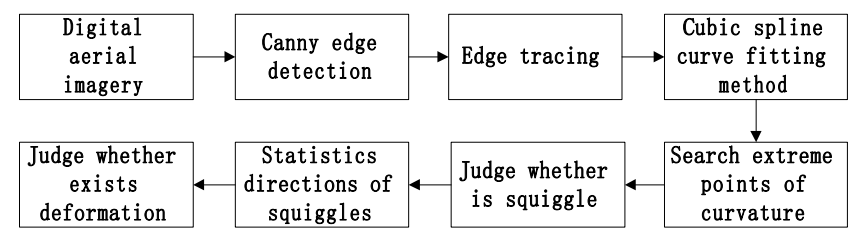

Figure 2. workflow of deformation inspection method for digital aerial imagery based on statistical characteristics

\subsection{Contour extraction and pre-processing of digital aerial imagery}

To find the squiggles, it is necessary to extract all the contours of images and judge each of them one by one. The contour extraction of image is composed of two steps: edge detection and contour tracing.

Currently, the common edge detection methods consist of Roberts operator, Sobel operator, Prewitt operator, Laplacian operator and Canny operator. Among them, Canny operator is based on the ideas of optimization and has the advantages of resistance to noise and accurate positioning. Therefore, it is adopted to detect the edges in this paper because of high accuracy of edge detection. The steps of the Canny edge detection are described as follows:

1) Smooth the image with Gaussian filter of specific standard deviations $\sigma$, thus reducing noise caused by light intensity, sensor's temperature, sensor's quality, etc;
2) For each pixel of the image, compute the amplitude and direction of the gradients through the finite difference of first order partial derivatives;

3) Conduct non-maximum suppression processing to identify edges. Partition the gradients according to the directions and search the local maximum pixels. Then set the value of the nonmaximum pixel zero in order to sharpen the edges of the image. 4) Judge the pixels using threshold $T_{1}$ and $T_{2}$, where $T_{1}<T_{2}$. The pixel with its value larger than $T_{2}$ is called strong edge pixel, and that between $T_{1}$ and $T_{2}$ is called weak edge pixel.

5) Set the weak edge pixels connected with the 8 neighborhoods of strong edge pixels as strong edge pixels, and then carry on binary processing based on whether the edge pixels are strong or not.

After acquiring the edges of the image, contour tracing process needs to be performed and the data of contour lines are saved in form of list to facilitate the location of squiggles.

The concrete steps of the contour tracing are described as follows:

1) Sequentially search all the pixels of the image from left to right and bottom to top, find out the boundary pixel at the bottom left corner and make it the start point.

2) From the first boundary point, the initial search direction is defined as left top. If the pixel at the left top is black, it is the boundary point. Otherwise, the direction will be rotated 45 degrees clockwise until the first black pixel appears.

3) Set the first black pixel as the new boundary point, the current search direction is rotated 90 degrees counterclockwise. Apply the same method to search the next black pixel until returning the original boundary point or no pixels are available. 4) Save the traced contours into the list and label them tracked.

5) Repeat the first step until the image has non-labeled contours.

The extracted contours cannot be applied directly into the graphic computing. The solution to this problem is to use smooth and derivable spline functions to fit the contours of the image. The fitting process of spline function is able to eliminate the noise for the contours of the image and makes it easier for the judgment of the squiggles of image at the same time.

Suppose the number of the pixels the current contour contains is $\mathrm{n}$. The functional relationship between the parameter $\mathrm{t}(\mathrm{t}=0,1$, $2, \ldots, n-1)$ and the plane curve formed by the contours of the current image is obtained with cubic spline curve fitting method, which is $c(t)=(x(t), y(t))$, where $x(t)$ is the function relationship between the $x$-axis of the plane curve and the parameter $t$, and $y(t)$ is the function relationship between the $y$-axis of the plane curve and the parameter $t$.

\subsection{Search and judgment of the squiggles in aerial imagery}

In this method, through searching the extreme point of curvature of each contour for the push-broom digital aerial imagery, the possible positions of squiggles are determined. After that, whether those points can constitute a squiggle or not will be figured out according to the location relationship between each extreme point of curvature and the surrounding points.

For the plane curve $c(t)=(x(t), y(t))$ given with parametric expressions, the calculation formula of the curvature $\mathrm{K}$ is shown in (1).

$$
K=\frac{\left|x^{\prime}(t) y^{\prime \prime}(t)-x^{\prime \prime}(t) y^{\prime}(t)\right|}{\left(x^{\prime 2}(t)+y^{\prime 2}(t)\right)^{3 / 2}}
$$


where $x^{\prime}(t)=$ first order derivative of the functional relationship between the $\mathrm{x}$-axis of the plane curve and the parameter $\mathrm{t}$

$y^{\prime}(t)=$ first order derivative of the functional relationship between the $y$-axis of the plane curve and the parameter $\mathrm{t}$

$x^{\prime \prime}(t)=$ the second derivative of the functional relationship between the $\mathrm{x}$-axis of the plane curve and the parameter $\mathrm{t}$

$y^{\prime \prime}(t)=$ second derivative of the functional relationship between the $y$-axis of the plane curve and the parameter $\mathrm{t}$

Apply derivation calculation to both sides of the formula of curvature and get the relationship between the first derivative $K^{\prime}$ of the curvature of the image contours and the parameter t.

$$
\begin{aligned}
& K^{\prime}=\frac{\left(x^{\prime}(t) y^{\prime \prime \prime}(t)-x^{\prime \prime \prime}(t) y^{\prime}(t)\right)\left(x^{\prime 2}(t)+y^{\prime 2}(t)\right)}{\left(x^{\prime 2}(t)+y^{\prime 2}(t)\right)^{5 / 2}} \\
& -\frac{-3\left(x^{\prime}(t) y^{\prime \prime}(t)-x^{\prime \prime}(t) y^{\prime}(t)\right)\left(x^{\prime}(t) x^{\prime \prime}(t)+y^{\prime}(t) y^{\prime \prime}(t)\right)}{\left(x^{\prime 2}(t)+y^{\prime 2}(t)\right)^{5 / 2}}
\end{aligned}
$$

where $x^{\prime \prime \prime}(t)=$ third order derivative of the functional relationship between the $\mathrm{x}$-axis of the plane curve and the parameter $\mathrm{t}$

$y^{\prime \prime \prime}(t)=$ third order derivative of the functional relationship between the $y$-axis of the plane curve and the parameter $\mathrm{t}$

If $K^{\prime}$ is 0 , the pixel corresponding to the parameter $\mathrm{t}_{0}$ is the extreme point of curvature in the contour of the image.

Since the power time of the parameter $t$ in the equation $K^{\prime}(t)=0$ is larger than 2, the formula of root cannot be used directly. Instead, only approximate values are determined with numerical method. The function $K^{\prime}(t)$ is smooth and derivable, and there exist both positive and negative values around the extreme points of curvature corresponding to the squiggles according to their characteristics. So the method of bisection can be used to solve the equation. General bisection methods can only find the simple root and odd multiple roots for the equation, but single image contour might have multiple different extreme points of curvature. For this reason, the bisection method needs to be adjusted and modified. The concrete steps after adjustment are as follows:

Suppose the number of the pixels the current processing contour contains is $\mathrm{n}, \mathrm{a}_{0}=0, \mathrm{~b}_{0}=\mathrm{n}$. For $\mathrm{k}=0,1, \ldots, \mathrm{m}$, perform:

1) compute $x_{k}=\frac{a_{k}+b_{k}}{2}$;

2) If $b_{k}-a_{k} \leq \varepsilon$ or $\left|f\left(x_{k}\right)\right| \leq \eta$, then set $s \approx x_{k}$ and record the approximate value $\mathrm{s}$ into the root list. Take the $\mathrm{k}$ from the list to be processed to replace the current $\mathrm{k} . \mathrm{x}_{\mathrm{k}}$ and $\mathrm{b}_{\mathrm{k}}$ taken from the list are defined as the processing regions $\left(a_{k}, b_{k}\right)$; otherwise turn to (3);

3) If $f\left(a_{k}\right) f\left(x_{k}\right)<0$ and $f\left(x_{k}\right) f\left(b_{k}\right)>0$, make $a_{k+1}=a_{k}$ and $b_{k+1}=x_{k}$; If $f\left(a_{k}\right) f\left(x_{k}\right)>0$ and $f\left(x_{k}\right) f\left(b_{k}\right)<0$, make $a_{k+1}=x_{k}$ and $b_{k+1}=b_{k}$; If $f\left(a_{k}\right) f\left(x_{k}\right)<0$ and $f\left(x_{k}\right) f\left(b_{k}\right)<0$, make $a_{k+1}=a_{k}$ and $b_{k+1}=x_{k}$. Then save the current $\mathrm{k}, \mathrm{x}_{\mathrm{k}}$ and $\mathrm{b}_{\mathrm{k}}$ into the list to be processed;

4) If $\mathrm{k}=\mathrm{m}$, output the unsuccessful iteration information $\mathrm{m}$ times. Take the $\mathrm{k}$ from the list to be processed to replace the current $\mathrm{k}, \mathrm{x}_{\mathrm{k}}$ and $\mathrm{b}_{\mathrm{k}}$ taken from the list are defined as the processing regions $\left(a_{k}, b_{k}\right)$; otherwise continue.

The parameter $t$ corresponding to each extreme point of curvature in the contours of image is figured out via the adjusted bisection method. However, the extreme points of curvature in the contours are not necessarily the vertexes of the squiggles, so it is essential to judge those extreme points one by one.

The waves formed from the squiggles of the digital aerial images possess certain amplitude and wavelength, which is comparatively obvious in the image. The judgment whether the extreme point and its surrounding points develop noticeable waves or not should on the basis of the characteristics of the squiggles. The method used in the paper is explained as follows: Suppose the number of the pixels in the contours which the extreme points of curvature located in is $n$, and the parameter corresponding to the current judging extreme point is $\mathrm{t}_{0}$, and the pre-set judging range of the surrounding points is $\left(\mathrm{t}_{0-1}, \mathrm{t}_{0+1}\right)$, where 1 is threshold for the surrounding points.

1) If $t_{0-1}<0$ or $t_{0+1}>n-1$, which indicates the extreme points are in the two ends, the number of the surrounding points is not enough to make up a squiggle and the judgment result is false; otherwise proceed to next step;

2) According to the function expression of fitting curve of the image contours, compute $\mathrm{x}$-axis and $\mathrm{y}$-axis of $\mathrm{P}_{0}, \mathrm{P}_{0-1}, \mathrm{P}_{0+1}$ in the contours corresponding to $\mathrm{t}_{0}, \mathrm{t}_{0-1}, \mathrm{t}_{0+1}$;

3) Compute the Euclidean distance between $\mathrm{P}_{0-1}$ and $\mathrm{P}_{0+1}$. If the value is smaller than threshold $T_{1}$, the judgment result is false; otherwise proceed to next step;

4) Compute the distance from $P_{0}$ to the segment $P_{0-1} P_{0+1}$. If the value is smaller than threshold $\mathrm{T} 2$, the judgment result is false; otherwise record $\mathrm{P}_{0}$ as the vertex of the squiggle;

Determine all the extreme points of curvature in the contours of the image according to the above steps and record all the vertexes of the squiggles.

\subsection{Statistical directions and distortion judgment for the squiggles of the aerial imagery}

The waves formed from the squiggles and their surrounding points of digital aerial images are not standard parabola. Consequently, the symmetrical directions of the waves cannot act as the opening directions of the waves the same way as parabola. But, the normal directions of the vertexes of the squiggles can well represent the directions of the squiggles; therefore, the normal direction of each vertex is determined as the direction of each squiggle.

According to the parametric equation of the squiggles of the image, compute the rad of the normal directions of the vertexes of the squiggles.

$$
r a d=\operatorname{atan}\left(-\frac{y^{\prime}(t)}{x^{\prime}(t)}\right)
$$

Turn the data rad in radian system into $\theta$ in degrees system, whose range is $[-180,180]$. When $x^{\prime}(t)=0, y^{\prime}(t)<0 \quad \theta=-180$; when $x^{\prime}(t)=0, y^{\prime}(t)>0 \quad \theta=180$; then save $\theta$ into the list. Compute each direction of each squiggle one by one according to the above steps. 
With the directions of all squiggles figured out, statistical analysis is carried out to inspect the deformation phenomenon of the image. Based on the common directions of the squiggles caused by deformation, divide the angle range of 0 to 360 degrees equally into eight intervals and every interval is 45 degrees. In the same distorted image, the directions caused by deformation usually appear with paired angles in the form of complementary angles. Therefore, those angles should be classified as the same characteristic when taking statistical analysis. To facilitate calculation, the threshold range of arc tangent is adopted as the statistical range, which is $[-180,180]$. Divide the statistical range into four direction intervals, respectively are $[-22.5,22.5],[22.5,67.5],[-67.5,-22.5]$ and [$90,-67.5] \cup[67.5,90]$, as is shown in the shadow region of the figure3.

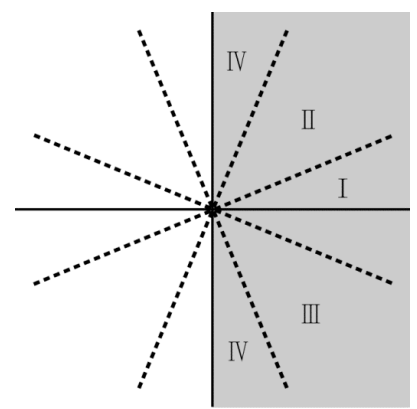

Figure 3. schematic of the intervals of statistical directions

Since the tangent values of the complementary angles are the same, the results of arc tangent are directly used as the data to be statistical analyzed regardless of the unit conversion of the angle data calculated by the formula (3). Count the number of squiggles in each direction interval, and then compute the judgment value $R_{\max }$ through dividing the maximum number with most squiggles by the total number. At last, whether there exists deformation or not is determined by comparing $\mathrm{R}_{\max }$ with the threshold $\mathrm{T}_{\mathrm{jud}}$. The pre-set $\mathrm{T}_{\mathrm{jud}}$, with the data range $(0.25,1)$, directly influences the judging results. The smaller the threshold $\mathrm{T}_{\text {jud }}$ is, the easier the deformation is to be judged.

\section{EXPERIMENTS AND RESULTS}

In the paper, 40 push-broom digital aerial images with the size $11000 \times 8000$ are selected as the experimental subjects, ten of which have deformation. Apply the deformation inspection method for digital aerial imagery based on statistical characteristics to these images, with the threshold $\mathrm{T}_{1}=6, \mathrm{~T}_{2}=6$, $\mathrm{T}_{\mathrm{jud}}=0.35$. The results are as follows: among the 30 images without deformation, 28 of them are inspected with no distortions and the left 2 images have distortions; among the 10 images with deformation, all of them are inspected with distortions. The average time of calculation of each image is $45 \mathrm{~s}$, and the accuracy rate of the inspection method is $95 \%$. The main reason for the two images with error results is that there exist distinct squiggles in the same directions in the geomorphic features of both images.

\section{CONCLUSIONS}

The deformation inspection method for digital aerial imagery based on statistical characteristics proposed in the paper achieved high accuracy and a relative high speed in the inspection of distortions caused by low accuracy of IMU data.
However, the texture contours of the images will directly affect the statistical results of the corresponding directions of the squiggles. So the discrimination between the squiggles used for deformation detection and the contours belonging to the image content will be one of the focuses in future studies. In addition, the different precise extent of IMU data will lead to some changes in the size of the squiggles of the images. As a result, the self-adaptation of thresholds based on actual images requires further researches in the order to realize automatic inspection.

\section{REFERENCES}

Lijun Zhang, Qiu Li, Yibin He, Dahai Guo, Yueguan Yan, 2006. Evaluation of GPS/ IMU supported aerial photogrammetry. International Geoscience and Remote Sensing Symposium (IGARSS), pp.1512-1514, 2006 IEEE International Geoscience and Remote Sensing Symposium, IGARSS.

Ballard D H, 1981. Generalizing the Hough transform to detect arbitrary shapes. Pattern Recognition, 13(2), pp. 111-122.

Rafael C. Gonzalez, Richar d E.Woods, 2004. Digital Image Processing. Trans. Qiuqi Ruan, Yuzhi Ruan, et al, Beijing: Publishing House of Electronics Industry of China.

H. Ming-Kuei, 1962. Visual pattern recognition by moment invariants. Information Theory, IRE Transactions, 8(2), pp.179187.

Xueyou Li, Rongjun Zhao, Yingcheng Li, Heping Lv, 2006. The application of IMU/DGPS-based photogrammetry in large scale map production. Science of Surveying and Mapping, 31 (1), pp. 60-61.

Kouibia A., Pasadas M, 2011. Rodriguez M. L. Optimization of parameters for curve interpolation by cubic splines. Journal of Computational and Applied Mathematics, 235(14), pp.41874198 .

\section{ACKNOWLEDGEMENTS}

The paper is supported by both State Basic Research Development Program ("863 Program") (2012AA12A305) and Major State Basic Research Development Program ("973 Program") (2009CB723905). 